\title{
Experiencias de largo plazo para el manejo de una hierba invasora de pastizales: El caso de Hieracium pilosella L. en la Estepa Fueguina
}

\author{
P.A. Cipriotti ${ }^{1}$; M.B. Collantes ${ }^{2}$; C. Escartín ${ }^{3}$; S. Cabeza $;$; R.B. Rauber ${ }^{5}$ \& K. Braun ${ }^{2}$ \\ 1. Dpto. de Métodos Cuantitativos y Sistemas de Información, Facultad de Agronomía - IFEVA, Universidad de Buenos Aires / \\ CONICET. Ciudad de Buenos Aires, Argentina. 2. Laboratorio de Ecología de Pastizales, Museo Argentino de Ciencias Naturales \\ - CONICET. 3. Departamento de Ecología y Ciencias Ambientales - CEBBAD, Universidad Maimónides. 4. Estancia Guazu-cue, \\ Tierra del Fuego. 5. Instituto Nacional de Tecnología Agropecuaria / CONICET, EEA Villa Mercedes, San Luis.
}

\begin{abstract}
RESUMEN. Las experiencias de largo plazo son poco frecuentes en los estudios sobre manejo de especies invasoras y resultan claves para comprender la efectividad de los controles. En este trabajo, revisitamos un experimento instalado hace más de siete años, para evaluar diferentes alternativas de control (inter-siembra de pasturas, fertilizaciones y aplicación de herbicidas selectivos y no selectivos) bajo dos condiciones de pastoreo (con y sin pastoreo) en pastizales de Tierra del Fuego invadidos por la maleza exótica Hieracium pilosella L. (velosilla, Asteraceae). Con el fin de evaluar los efectos de los tratamientos aplicados hace siete años, se midió la cobertura de la especie invasora, de las formas de vida dominantes y del suelo desnudo. Los efectos de la fertilización dependieron de las condiciones de pastoreo; la cobertura de $H$. pilosella luego de siete años disminuyó en más de un $82 \%$ y fue reemplazada por otras hierbas (mayormente especies naturalizadas y de valor forrajero) en las parcelas fertilizadas y sin pastoreo doméstico, mientras que no hubo cambios en las parcelas fertilizadas y pastoreadas. Por otro lado, ambos herbicidas (selectivos y no selectivos) redujeron la cobertura de $H$. pilosella en ca. $90 \%$ luego de siete años, independientemente del pastoreo. Sin embargo, la aplicación del herbicida no selectivo determinó un aumento de la cobertura de suelo desnudo de hasta un $15 \%$. Con la aplicación del herbicida selectivo, por el contrario, la cobertura de graminoides aumentó hasta casi un $60 \%$ y la cobertura de suelo descubierto se mantuvo por debajo del $2 \%$. Estos resultados permiten sugerir que una estrategia de control basada en la aplicación local de herbicidas selectivos y/o fertilizaciones con exclusiones temporarias del ganado ovino reduce la cobertura de la maleza invasora a escala local y a mediano plazo.
\end{abstract}

[Palabras clave: control químico, disturbios, invasiones, manejo del pastoreo, pastizales, pastoreo ovino, pasturas]

\begin{abstract}
АвsтRAст. Long-term experiences for the management of a grassland invasive herb: The case of Hieracium pilosella L. in the Fuegian Steppe: Long-term experiences are generally rare in studies on management of invasive species, and they are the key to understand the effectiveness of controls. In this work, we revisited a field experiment set seven years ago to evaluate the effects of four control measures (pasture sown + fertilizer, fertilizer and selective/non-selective herbicide applications) under two different grazing conditions (grazed and ungrazed) in rangelands from northern grasslands of Tierra del Fuego Island in Southern Patagonia (Argentina) invaded by the exotic weed Hieracium pilosella L. (mouse-ear hawkweed, Asteraceae). To evaluate the effects of the treatments applied seven years ago, we measured the cover of the invasive species, the dominant growth forms, and bare soil. The effects of fertilization depended on the grazing conditions; H. pilosella cover decreased by more than $82 \%$ and it was replaced by naturalized herbs with high forage value in the ungrazed-fertilized plots, while it exhibited no decrease in the grazed-fertilized plots after seven years since the application. Both herbicides (selective and non-selective) reduced H. pilosella cover by ca. $90 \%$ independently of grazing. However, the non-selective herbicide application resulted in an increase in bare soil cover (15\%) independently of grazing. In contrast, the selective broadleaf herbicide application increased the graminoids cover up to a $60 \%$ and the bare soil cover was below the $2 \%$. According with the results, we suggest a control strategy based on the local application of selective herbicides and/or fertilizers in conjunction with a transient ban on sheep grazing because it is able to reduce the invader's cover at midterm and local scale.
\end{abstract}

[Keywords: chemical control, disturbances, invasions, grasslands, grazing management, pastures, rangelands, sheep grazing]

\section{INTRODUCCIÓN}

El crecimiento de la población humana y el desarrollo de las sociedades modernas en los últimos siglos han generado una presión sobre los recursos naturales a nivel global ( $\mathrm{Di}$
Castri et al. 1989). Esto trajo aparejado cambios complejos en las actividades productivas, con innovaciones tecnológicas impensadas hace un siglo que permitieron incrementar la producción. Sin embargo, frecuentemente se han documentado consecuencias indeseables Última versión: 13 de noviembre; Aceptado: 16 de noviembre. 
sobre el medioambiente. Ejemplos que se repiten a nivel planetario involucran procesos de contaminación, deforestación, desertificación, pérdida de diversidad biológica, cambios climáticos e invasiones biológicas (Mack et al. 2000; Schlesinger et al. 1990; Vitousek et al. 1997; Reynolds et al. 2007).

Los pastizales son ecosistemas particularmente susceptibles a las invasiones biológicas debido a la implantación de cultivos o la introducción de ganado doméstico (Mack 1989; Hobbs \& Huenneke 1992; Buckland et al. 2001). El pastoreo puede promover la introducción y expansión de especies de plantas exóticas debido a la alimentación selectiva, al pisoteo, o al aumento en la distancia máxima de dispersión de semillas por parte de los herbívoros (Brown \& Archer 1989; Bellingham \& Coomes 2003). Además, el laboreo del suelo asociado con la implantación de pasturas y otras prácticas puede facilitar la colonización de malezas exóticas (Hobbs \& Huenneke 1992; Davis et al. 2000; Walker et al. 2005). La presencia de malezas invasoras generalmente reduce la calidad y cantidad de forraje disponible para el ganado, incrementa los costos de manejo y puede perjudicar la respuesta de los animales (DiTomaso 2000).

La ganadería ovina extensiva es una característica sobresaliente de los ecosistemas pastoriles Patagónicos desde hace más de 120 años (Soriano 1983; Aagesen 2000; Anchorena et al. 2001). Dicha actividad provee de lana y carne para mercados locales e internacionales, contribuyendo al desarrollo de la región junto a otras actividades económicas como la petrolera, forestal, minera, pesca y turismo. El principal recurso natural comprometido en la ganadería es el de los pastizales, que han sufrido distintos procesos de degradación debido principalmente al manejo inadecuado del pastoreo doméstico (Paruelo et al. 1993; Perelman et al. 1997). Entre los principales problemas podemos mencionar: erosión y salinización del suelo, arbustización, reemplazos de especies palatables por menos palatables, e invasiones biológicas que pueden llevar a una caída en la receptividad ganadera (Golluscio et al. 1998; Speziale \& Ezcurra 2011). En este contexto, la conservación de los pastizales naturales es clave para asegurar, al menos desde la dimensión bio-física, la sustentabilidad de la actividad ganadera en la región.
La invasión de Hieracium pilosella L. (Asteraceae) en la Patagonia Austral (Tierra del Fuego y Santa Cruz en Argentina y Región de Magallanes en Chile; Domínguez 2004; Livraghi et al. 1998) representa una nueva amenaza, ya que se trata de una especie invasora exitosa en pastizales neocelandeses (Allan 1924), norteamericanos (Voss \& Böhlke 1978) y dentro de su propia área de origen (Winkler \& Stöcklin 2002). Esta hierba perenne es originaria de los pastizales del norte de Europa y Asia occidental (Bishop \& Davy 1994). Es una especie altamente competitiva y se propaga rápidamente por la formación de parches mono-específicos a través del crecimiento vegetativo de estolones y rizomas (Moen \& Meurk 2001). Su principal impacto económico es una disminución de la biomasa forrajera y de la productividad secundaria causada por la sustitución de especies nativas y palatables de los pastizales originales (Makepeace 1985a; Makepeace et al. 1985; Treskonova 1991; Johnstone et al. 1999). En Tierra del Fuego (Argentina), la región invadida actual está también dominada por pastizales de festuca (Festuca gracillima Hooker f.) y matorrales de mata negra fueguina (Chiliotrichum diffusum (Forster f.) O. Kunze) (Livraghi et al. 1998; Cipriotti et al. 2010). En la actualidad, la invasión de $H$. pilosella se haya muy extendida en la región de la estepa, donde comúnmente reemplaza a las especies nativas del inter-coironal o los inter-espacios entre arbustos. Estos sitios son naturalmente ocupados por un gran conjunto de gramíneas nativas pequeñas y especies naturalizadas que representan un aporte clave a la diversidad y al valor pastoril de esa comunidad (Posse et al. 1996), y por lo tanto su preservación resulta fundamental para la conservación de la biodiversidad y la receptividad ganadera.

Dado los problemas ocasionados por esta especie en otros pastizales del mundo en las décadas del ' 60 y' 70 , existen muchos trabajos sobre alternativas para su control. Entre las más estudiadas se destacan el control químico mediante el uso de herbicidas y fertilizantes (Scott 1993; Woodman et al. 1997; Norton et al. 2006), y el control biológico (Grosskopf et al. 2002; Mansilla et al. 2011). En general, las medidas apropiadas para contener y controlar las invasiones de malezas buscan promover la regeneración de las especies nativas, mediante la alteración del régimen de perturbaciones y/o la modificación de la disponibilidad de recursos entre las especies dentro de la comunidad receptora (Huwer et al. 2005; 
Firn et al. 2008, 2010). En la estepa Fueguina durante 2005 llevamos adelante un estudio sobre alternativas de control para mitigar los efectos de la maleza mediante inter-siembras, aplicación de fertilizantes y herbicidas en combinación con la exclusión del ganado doméstico basados en experiencias similares en el mundo y la región. Los resultados a corto plazo de los controles fueron muy alentadores, a pesar de que ninguno logró una erradicación local de la especie (Cipriotti et al. 2012). El objetivo principal de este trabajo es cuantificar la respuesta a cuatro alternativas de control y manejo pastoril mediante una reevaluación de un ensayo instalado hace más de siete años. En segundo lugar, se presenta y discute una propuesta de manejo para el estado actual de la invasión de $H$. pilosella en la estepa Fueguina, basada principalmente en los resultados actuales y lo que se sabe sobre el control de esta especie en otros sitios.

\section{MÉTOdOS}

\section{Sitio de estudio}

El experimento a campo se estableció en la Estancia Cullen, ubicada al norte de la estepa Fueguina $\left(52^{\circ}\right.$ $55^{\prime} \mathrm{S}, 68^{\circ} 33^{\prime} \mathrm{W}$ ), dentro de un potrero (ca. 1220 ha) con un sector utilizado para la implantación de pasturas hace 30 años y con una cobertura actual promedio de la especie invasora mayor al 15\% (Cipriotti et al. 2012). La precipitación media anual medida cerca del casco de la estancia es de $335 \mathrm{~mm}$ / año (últimos 100 años), mientras que la temperatura media del mes más frío es $0^{\circ} \mathrm{C}$ y alcanza los $10^{\circ} \mathrm{C}$ en el mes más cálido (datos tomados en la ciudad de Río Grande, 53 $47^{\prime} 27^{\prime \prime}$ S, $\left.67^{\circ} 42^{\prime} 46^{\prime \prime} \mathrm{W}\right)$. La vegetación dominante de la estepa Fueguina fue clasificada como una estepa graminosa húmeda (Collantes et al. 1999, 2005). Las zonas altas son dominadas por una estepa de matas cespitosas de Festuca gracillima Hooker f. que alternan con matorrales dominados por Chiliotrichum diffusum (Forster f.) O. Kunze o murtillares dominados por Empetrum rubrum Vahl ex Willd en función de la humedad y del tipo y grado de acidez de los suelos. Las zonas más bajas son dominadas por vegetación hidrófila. En el potrero seleccionado para el estudio, los arbustos de mata negra (C. diffusum) fueron eliminados por medios mecánicos y fuego durante la década de 1980, para luego sembrar especies forrajeras (por ejemplo, Festuca arundinacea Schreb., Dactylis glomerata L., Trifolium repens L.) con el fin de mejorar la calidad del pastizal para la cría extensiva de ganado ovino.

\section{Experimento}

En octubre de 2005 se estableció el experimento dentro del potrero antes descripto. Se seleccionaron seis áreas homogéneas de aproximadamente 1 ha (parcelas) que contuvieran aproximadamente al menos 100 parches de $H$. pilosella de aproximadamente $2 \mathrm{~m}$ de diámetro. Tres de las seis parcelas delimitadas fueron seleccionados al azar y excluidas al ganado ovino mediante la construcción de un cerco permanente de cinco alambres de $1.4 \mathrm{~m}$ de altura con el distanciamiento diferencial entre alambres para evitar el paso de las ovejas. De este modo, se consideraron a las tres parcelas abiertas al pastoreo doméstico pertenecientes al tratamiento "pastoreo" y a las tres parcelas excluidas al ganado ovino correspondientes al tratamiento "clausura". Antes del establecimiento del experimento, las seis parcelas (con y sin pastoreo) tuvieron un manejo y carga animal similar durante las últimas décadas. Además, todas las parcelas tenían una posición topográfica, pendiente del terreno, cobertura vegetal y composición florística similar, incluyendo la cobertura de la maleza exótica $H$. pilosella (ca. $35 \%)$.

Dentro de cada parcela se establecieron cinco sub-parcelas $(5 \times 5 \mathrm{~m})$ asegurando por lo menos la existencia de un parche de $H$. pilosella (diámetro aprox. $2 \mathrm{~m}$ ), lo que resultó en un total de $30 \mathrm{sub}$ parcelas. Para caracterizar la situación inicial antes de la aplicación de los tratamientos, se midió la cobertura de $H$. pilosella y las formas de vida dominantes (i.e. gramíneas, ciperáceas, arbustos, arbustos enanos y hierbas dicotiledóneas) en una transecta permanente oblicua a través del centro de la sub-parcela de acuerdo con Cingolani et al. (2005). Además, se tomaron fotos y dibujaron mapas con la ubicación y la forma aproximada de todos los parches de $H$. pilosella en cada sub-parcela.

Cuatro tratamientos de control y un testigo sin tratar fueron asignados al azar a las sub-parcelas, luego de la aleatorización inicial referida a la condición de pastoreo (parcelas): (1) Testigo sin tratar; (2) Inter-siembra de pastura (10:5:5 kg.ha1, Dactylis glomerata : Trifolium repens : Trifolium pratense) + fertilización NP con fosfato diamónico (200 kg.ha $\left.{ }^{-1}, 18-46\right) ;$ (3) Fertilización NP con fosfato diamónico (200 kg.ha-1 , 18-46); (4) Aplicación de herbicida selectivo de hoja ancha [2,4-DB éster (93\% p.a.) en $\left.1.51 \cdot \mathrm{ha}^{-1}\right]$; y (5) Aplicación de herbicida no selectivo [Glifosato@ (48\% p.a.) en $11 \cdot \mathrm{ha}^{-1}$ ]. Los tratamientos de fertilización e inter-siembra de especies forrajeras tuvieron por objeto mejorar la vegetación nativa o naturalizada, mientras que las aplicaciones de herbicidas se centraron en la eliminación de la maleza. La mezcla de especies de gramíneas y leguminosas forrajeras seleccionadas para el experimento es utilizada en la región para mejorar la calidad de los pastizales en pastoreo (ver detalles de aplicación en Cipriotti et al. 2012). Al inicio de la segunda estación de crecimiento (octubre 2006), se repitió la aplicación de los fertilizantes y herbicidas a las sub-parcelas respectivas, siguiendo el mismo protocolo del primer año. Las variables de respuesta estudiadas al final de la séptima estación de crecimiento (2013) 
fueron la cobertura de la maleza, la cobertura de las formas de vida dominantes (sensu Cingolani et al. 2005) y la cobertura de suelo descubierto. Todas las mediciones de cobertura se realizaron utilizando el método de intercepción lineal a lo largo de una transecta diagonal que cruzaba toda la sub-parcela.

\section{Análisis estadísticos}

El experimento se basó en un diseño en parcelas divididas con la parcela principal utilizada para determinar el efecto del pastoreo doméstico (dos niveles: con y sin pastoreo) y las sub-parcelas utilizadas para evaluar las alternativas de control (cinco niveles de acuerdo con los tratamientos ya descriptos). Se realizó un análisis de varianza para un modelo de parcelas divididas con interacción, con las sub-parcelas dentro de las parcelas principales, con el pastoreo, las alternativas de control y su interacción como los únicos efectos fijos y el error como el único término aleatorio del modelo. Al detectar efectos significativos para algún término del modelo en el análisis de varianza, se realizaron pruebas post-hoc para los diferentes tratamientos según la prueba LSD de Fisher con un nivel de significancia (alfa) del 5\%.

\section{RESULTADOS}

Luego de siete años de la aplicación de los tratamientos se detectaron efectos de los tratamientos que dependieron de la condición de pastoreo sobre la cobertura de la especie invasora y la de otras dicotiledóneas herbáceas. En cambio, las diferencias observadas en la cobertura de graminoides y de suelo desnudo solo obedecieron a cambios en las práctocas de manejo (Tabla 1).

La fertilización redujo un 85\% la cobertura de la $H$. pilosella solo en las parcelas sin pastoreo doméstico (Figura 1a). Esta disminución fue acompañada por un aumento en la cobertura de hierbas dicotiledóneas mayor al 100\% (Tabla 1; Figura 1b), debido principalmente a la respuesta del trébol blanco (Trifolium repens L.), diente de león (Taraxacum officinale Weber ex F.H. Wigg.) y vinagrillo (Rumex acetosella L.) (datos no publicados).

Ambos herbicidas redujeron en el mediano plazo la cobertura de $H$. pilosella al 3.9\%, lo que representa la décima parte del promedio en las sub-parcelas testigo no tratadas, independientemente del pastoreo doméstico, y alcanzando un nivel de control no diferenciable estadísticamente de la fertilización y exclusión del pastoreo doméstico (Figura 1a). Sin embargo, los diferentes herbicidas difirieron en sus efectos sobre la cobertura de dicotiledóneas, graminoides y suelo desnudo a
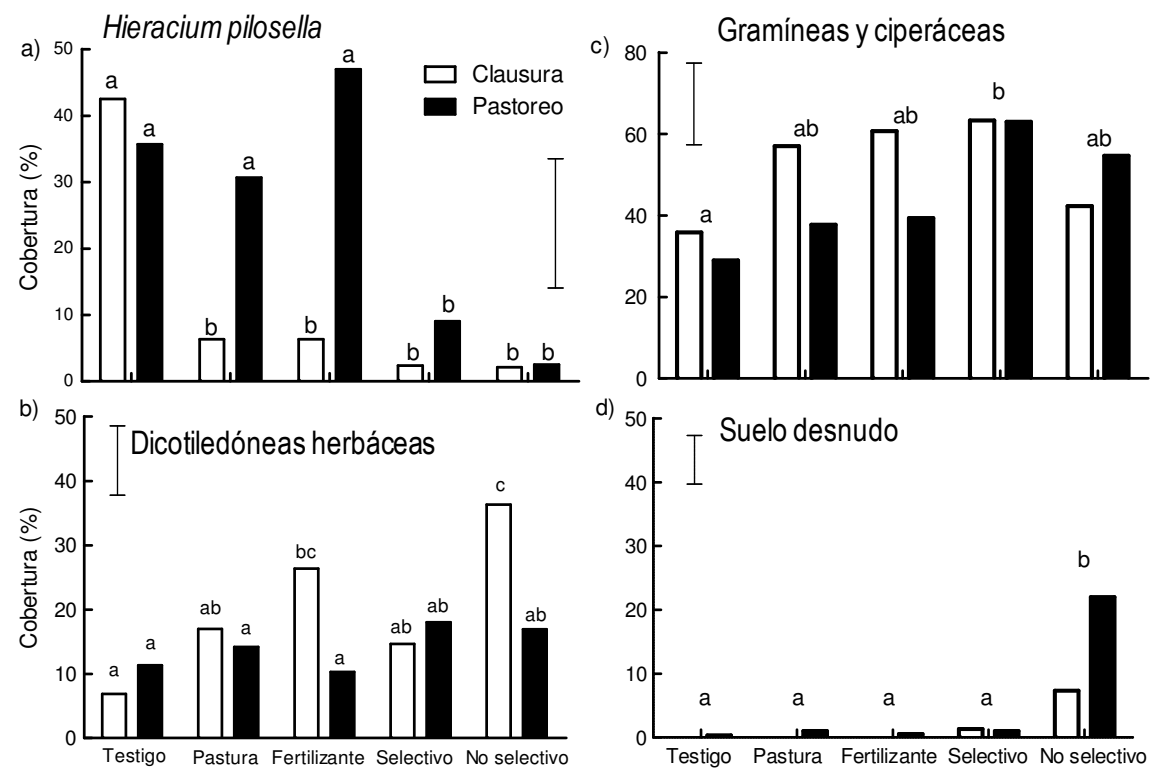

Figura 1. Cobertura promedio de los componentes de la vegetación analizados y del suelo desnudo al cabo de siete años de la aplicación de distintos tratamientos en parcelas pastoreadas y clausuradas al pastoreo. Las barras indican las medias y letras distintas indican diferencias estadísticamente significativas mediante pruebas post-hoc LSD (alfa=5\%). El segmento vertical representa la diferencia minima significativa para la prueba realizada.

Figure 1. Mean percent cover of plant components and bare soil under different treatments in ungrazed and grazed plots after the seven years of the experiment beginning. Wide bars indicate means and different letters indicate statistically significant differences according to post-hoc LSD tests (alpha=5\%). The hated segment for each panel represents the least significant difference for the respective statistical test. 
Tabla 1. Resumen de los análisis de varianza realizados para las distintas variables de respuesta estudiadas al cabo de siete años de aplicados los tratamientos de control (Manejo) y la condición de pastoreo (Pastoreo).

Table 1. Summary of ANOVAs for the different response variables here analyzed after seven years of the experiment beginning for control treatments (Management) and grazing condition (Grazing).

\begin{tabular}{lccccr}
\hline Variable respuesta & $\begin{array}{c}\text { Fuente } \\
\text { variación }\end{array}$ & g.l. & CME & F & valor-P \\
\hline Cobertura de & Pastoreo & 1 & 1276.62 & 8.38 & $\mathbf{0 . 0 1 1}$ \\
H. pilosella & Manejo & 4 & 1376.23 & 9.04 & $<\mathbf{0 . 0 0 1}$ \\
& Px M & 4 & 557.29 & 3.66 & $\mathbf{0 . 0 2 7}$ \\
Cobertura de hierbas & Error & 16 & 152.33 & & \\
dicotiledóneas & Pastoreo & 1 & 277.25 & 5.99 & $\mathbf{0 . 0 2 6}$ \\
& Manejo & 4 & 238.79 & 5.17 & $\mathbf{0 . 0 0 7}$ \\
& Px M & 4 & 182.41 & 3.95 & $\mathbf{0 . 0 2 1}$ \\
Cobertura de pastos & Error & 16 & 46.22 & & \\
graminoides & Pastoreo & 1 & 620.17 & 3.01 & 0.102 \\
& Manejo & 4 & 720.74 & 3.49 & $\mathbf{0 . 0 3 1}$ \\
& P x M & 4 & 427.72 & 2.07 & 0.133 \\
Cobertura de & Error & 16 & 206.55 & & \\
suelo desnudo & Pastoreo & 1 & 78.73 & 2.18 & 0.159 \\
& Manejo & 4 & 240.89 & 6.66 & $\mathbf{0 . 0 0 2}$ \\
& Px M & 4 & 61.55 & 1.70 & 0.199 \\
& Error & 16 & 36.18 & & \\
\hline
\end{tabular}

siete años de iniciado el control. La aplicación del herbicida no selectivo determinó un aumento mayor al $100 \%$ en la cobertura de dicotiledóneas herbáceas solo en las parcelas clausuradas (Figura 1b) y un aumento de más de cinco veces en la cobertura de suelo desnudo (llegando hasta casi el 15\%) en comparación con el testigo e independientemente de la condición de pastoreo (Figura 1d). Las subparcelas tratadas con el herbicida selectivo, por el contrario, exhibieron un aumento de un $90 \%$ en la cobertura de los graminoides diferenciándose significativamente del testigo (Tabla 1; Figura 1c) y una cobertura casi nula de suelo desnudo (Figura 1d), ambos efectos independientes de la condición de pastoreo (Tabla 1).

\section{DISCUSIÓN}

\section{Efectividad a largo plazo de las alternativas de control de la invasión}

La re-evaluación del experimento a campo de las alternativas de control de $H$. pilosella (fertilización con y sin inter-siembra combinados con manejo pastoril, y distintos herbicidas), mostró que los tratamientos fueron efectivos en el mediano plazo, ya que la disminución marcada de la cobertura de la especie invasora se mantuvo luego de siete años. Sin embargo, los tratamientos de control y manejo del pastoreo difirieron en su impacto sobre la vegetación nativa y naturalizada y sobre el ecosistema, en particular la cobertura de suelo desnudo.

Los resultados demostraron que las fertilizaciones en conjunción con la exclusión del pastoreo doméstico permitieron exaltar la respuesta de la vegetación nativa y naturalizada del inter-coironal, promoviendo un descenso significativo de la cobertura de $H$. pilosella luego de siete años del control inicial. Estos resultados coinciden con lo observado a corto plazo sobre la reducción de la cobertura de la invasora para el mismo experimento (Cipriotti et al. 2012). Además, la comparación con las respuestas de la invasora al año del a) clausura

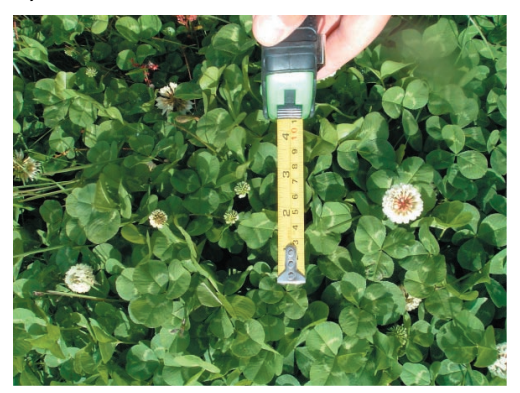

b) pastura

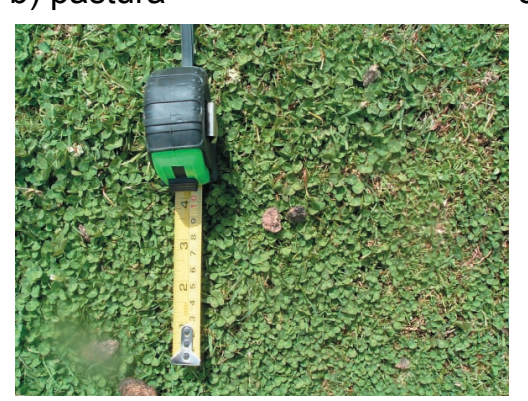

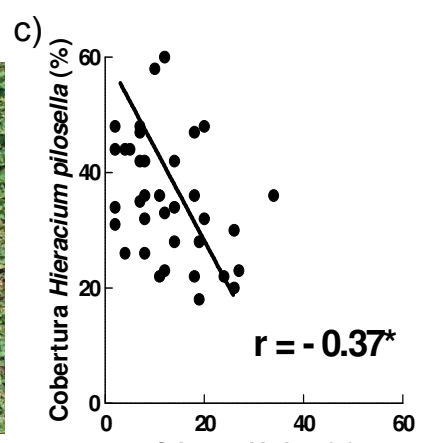

Cobertura hierbas $(\%)$

Figura 2. Manchones de trébol blanco (Trifolium repens L.) en las sub-parcelas fertilizadas con $200 \mathrm{kgxha}^{-1}$ de fosfato diamónico luego de dos años de la aplicación y con precipitaciones mayores al promedio durante la estación de crecimiento en parcelas clausuradas (a) y pastoreadas (b). c) Correlación entre la cobertura de la maleza H. pilosella y la cobertura de hierbas dicotiledóneas adaptado de un estudio descriptivo previo realizado a escala regional (Rauber et al. 2012).

Figure 2. Detailed photos of white clover spots (Trifolium repens L.) in the fertilized sub-plots with $200 \mathrm{kgxha}^{-1}$ of di- $^{-}$ ammonic phosphate after two years of application and with high rainfalls in ungrazed (a) and grazed (b) plots. c) Correlation between the cover of $H$. pilosella and the cover of dicotyledonous herbs from a descriptive study at regional scale (Rauber et al. 2012). 
control muestra que la recuperación de $H$. pilosella bajo la combinación de exclusión del pastoreo y fertilización N-P es relativamente lenta, ya que luego de siete años la cobertura no superó el 8\%. Experiencias de control de distinto tipo (e.g., manejo, químico, biológico) abundan para esta especie exótica en la bibliografía específica y en la mayoría de los casos, los resultados indican que la cobertura de la especie exótica reduce su cobertura en el corto y largo plazo mediante el manejo intensivo de los pastizales (Scott 1993; Scott \& Sutherland 1993; Woodman et al. 1997; Lamoureaux et al. 2003; Norton et al. 2006). Sin embargo, no se habían documentado hasta el momento efectos interactivos entre las medidas de control comúnmente empleadas (e.g., fertilizantes, herbicidas, intersiembra de pasturas, etc.) y el manejo del pastoreo (Scott 1993; Rose et al. 1995; Duncan et al. 1997; Meurk et al. 2002; Walker et al. 2005; Rose \& Frampton 2007).

La naturaleza de la respuesta interactiva observada en este estudio puede obedecer en parte al estadío de la invasión, ya que durante las fases tempranas de la invasión la cobertura de la maleza es baja y el inter-coironal está mayormente ocupado por un conjunto de especies deseables y diversas (i.e. graminoides nativas pequeñas y dicotiledóneas herbáceas nativas o naturalizadas; Posse et al. 1996), con lo cual el pastoreo selectivo puede reducir la intensidad de competencia entre la maleza y las especies palatables. En este contexto, el agregado de nutrientes y la exclusión del pastoreo, puede promover el crecimiento de competidores ya establecidos, resultando en un aumento rápido en la altura del canopeo y afectando la supervivencia de la maleza principalmente por la competencia por luz, como ya se ha visto para $H$. pilosella en estudios controlados en invernáculo (Grime \& Jeffrey 1965). Estudios correlativos a partir de censos de vegetación en la región de la estepa apoyan esta relación (Figura 2; Rauber et al. 2012). Sin embargo, en estadíos avanzados de la invasión donde la maleza es la única especie dominante en parches mono-específicos muy grandes (> $10 \mathrm{~m}$ de diámetro) y donde prácticamente no quedan especies del inter-coironal, esta medida de control claramente no podría favorecer a ninguna especie competidora a escala local.

Ambos herbicidas evaluados fueron muy efectivos en reducir la cobertura de $H$. pilosella en el mediano plazo, a pesar de que no se logró la erradicación local de la maleza. Sin embargo, los herbicidas variaron en sus efectos sobre la cobertura de otros grupos funcionales y del suelo desnudo. El herbicida selectivo, determinó un aumento de la cobertura de graminoides aún luego de siete años, lo que era esperable dada la naturaleza de su principio activo, pero no modificó la cobertura del suelo desnudo manteniéndose en niveles muy bajos ( $<2 \%)$. Estos resultados coinciden con los observados a corto plazo, luego de uno y dos años de la aplicación de los controles (Cipriotti et al. 2012). En cambio, la aplicación del herbicida de acción total en las parcelas clausuradas aumentó mucho la cobertura de dicotiledóneas herbáceas al cabo de siete años, mientras que la cobertura de graminoides no se diferenció del testigo. Esta respuesta es indicativa de la recuperación del impacto inicial del herbicida no selectivo en los primeros años, debido a la sucesión natural propia de estas comunidades donde las especies pioneras son $R$. acetosella, $T$. officinale y P. pratensis (Collantes et al. 2005) y probablemente a la mayor disponibilidad de semillas dentro de las áreas clausuradas. Por otra parte, la cobertura de suelo desnudo en las parcelas tratadas con el herbicida no selectivo fue la más alta, alcanzando casi un 15\% e independiente de la ocurrencia de pastoreo. Esta respuesta, claramente se diferenció de lo hallado en la evaluación a corto plazo, donde la cobertura de suelo desnudo fue mucho más alta $(>40 \%)$ y claramente superior en las parcelas pastoreadas por acción del pisoteo (Cipriotti et al. 2012). Si bien luego de siete años la comunidad presentó una recuperación importante aún bajo pastoreo en términos de la mayor cobertura de gramíneas y dicotiledóneas pioneras, la existencia aún de un $15 \%$ de cobertura de suelo desnudo ubica al uso de herbicidas no selectivos dentro de prácticas desaconsejables, en especial para áreas invadidas de gran extensión.

\section{Implicancias para el manejo de la invasión}

La efectividad de las alternativas de control de la invasión de $H$. pilosella evaluadas en este estudio queda supeditada a cambios de contexto dentro de la complejidad del agro-ecosistema ganadero de la región. En este sentido, cabe señalar principalmente a los cambios en las condiciones climáticas y ecológicas (de la invasión y de las comunidades recipientes), las diferencias de escala espacial, los costos económicos de las distintas prácticas, y la logística y capacidad 
de gestión de los distintos establecimientos de la zona. Por otro lado, no caben dudas que las prácticas a nivel predial deberían ser acompañadas e integradas en planes de manejo a escala regional de la invasión que involucren a los distintos actores (Simberloff 2009).

La efectividad de las fertilizaciones con nitrógeno y fósforo en combinación con la exclusión temporal del ganado puede verse sensiblemente afectada en función del contexto en la que se aplica, sobre todo del estado de la comunidad vegetal invadida (recipiente) y de las condiciones climáticas. Una comunidad con una cobertura muy alta de la invasora puede no responder al tratamiento por la falta de respuesta de la vegetación nativa y naturalizada. En este contexto, el fertilizante solo sería utilizado por la especie invasora, al igual que fertilizar sin remover el pastoreo. Por otro lado, la precipitación es clave, ya que la humedad durante la estación de crecimiento (octubre-febrero) permite mejorar el efecto de los fertilizantes y aumentar el crecimiento de las especies competidoras, en especial del trébol blanco (Figura 2). El trébol blanco es una especie muy demandante de agua y también una de los mejores competidoras de H. pilosella (Scott $\&$ Sutherland 1993). En este estudio, durante la aplicación de la primera fertilización (2005), las precipitaciones al comienzo (noviembre) y a mediados (enero) de la estación de crecimiento fueron particularmente altas con valores que superaron en un $287 \%$ y $157 \%$ las medias históricas mensuales, exaltando seguramente el efecto de los fertilizantes.

Otro aspecto importante a considerar a la hora de extrapolar estos resultados es la escala espacial de los ensayos aquí presentados y la escala espacial real de las áreas invadidas. En este sentido, las tecnologías de fertilización o pulverización aquí empleadas fueron posibles dado el tamaño pequeño de las parcelas experimentales $\left(25 \mathrm{~m}^{2}\right)$, sin embargo a la hora de pulverizar un potrero de cientos de hectáreas esta técnica no resultaría la más eficiente a priori por varias razones, entre las principales la capacidad de las mochilas portátiles (15-251). Sin embargo durante 2011, Cabeza y colaboradores realizaron un ensayo donde se evaluó una estrategia de control que integraba las aquí presentadas con excepción del manejo pastoril (i.e. herbicida 2,4 DB, más inter-siembra de forrajeras y fertilización $\mathrm{N}$ $P$ en distintos momentos del año) con un equipamiento apropiado para abordar las extensiones típicas a nivel de potrero en esta región (cuatriciclo $4 \times 4$ con cisterna y botalón incorporado para la pulverización) y sobre distintas comunidades vegetales con estructuras del canopeo muy disímiles (i.e. céspedes, coironales y matorrales), obteniendo valores finales de cobertura la maleza entre el $5-15 \%$ a pesar de valores iniciales superiores al $45 \%$ en todos los casos.

En el mismo trabajo los autores analizaron la factibilidad técnica y económica del manejo propuesto. De allí surge que las condiciones climáticas, sobre todo para aplicar el herbicida, resultan críticas para alcanzar el éxito de la práctica condicionado fundamentalmente por la temperatura y la velocidad del viento. Ambos factores reducen la ventana de aplicación a momentos muy estrechos que sumados a la restricción fenológica para que el control ocurra previo a la floración de la maleza, la aplicación resultaría viable solo durante algunos días entre octubre y diciembre (Cabeza et al. 2011). Esto podría considerarse una limitante importante para el planeamiento de la actividad y la organización de las tareas dentro de las estancias al superponerse con otros momentos de alta demanda de personal como la esquila.

Experiencias realizadas en pastizales similares en la región Magallánica de Chile con el herbicida Picloram $\odot$ o con distintas mezclas incluyendo 2,4 DB mostraron también resultados muy efectivos en el corto plazo para el control de esta maleza, acompañado en algunos casos de un "rejuvenecimiento" de las matas cespitosas de F. gracillima (Salinas Breskovic 2002; Strauch Bertin 2012). Las ventajas del Picloram $(\subset$ obedecen a la mayor residualidad del principio activo en el suelo y por lo tanto un aumento en la ventana temporal del control, mientras que una desventaja sería la mayor susceptibilidad del trébol blanco y otras hierbas deseables a este herbicida en comparación con el 2,4 DB (Salinas Breskovic 2002). La aplicación de un herbicida de mayor residualidad como el Picloram (C) condiciona el manejo a futuro de los sitios controlados durante un periodo más largo de tiempo, sobre todo si se piensa en inter-siembras.

Respecto del análisis económico, el trabajo de Cabeza et al. (2011) estudia pormenorizadamente los costos de esta práctica y muestra que la aplicación de herbicidas es en general accesible a los 
productores en relación con el costo, aunque el principal condicionante es el acceso a la maquinaria para las pulverizaciones (p.ej. pulverizadoras autopropulsadas). En cambio, los fertilizantes tienen un costo mayor y resulta difícil conseguir las cantidades requeridas. No obstante, estos estudios económicos no tuvieron en cuenta los beneficios directos e indirectos generados por la práctica del control de esta maleza invasora y sólo se centraron en un análisis de los costos.

El mantenimiento de la cobertura del suelo es un tema de vital importancia en pastizales sub-húmedos como los del norte de la Estepa Fueguina con el fin de evitar la erosión del suelo, la degradación del pastizal y la pérdida de productividad primaria y secundaria (Anchorena et al. 2001; Collantes et al. 2005). Es por esto, que en áreas muy invadidas y de gran extensión, cualquier práctica de remoción de la maleza (química o mecánica) debería ir acompañada de prácticas de recuperación de la cobertura vegetal para no dejar el suelo expuesto. Forzosamente, uno de los objetivos de las medidas de prevención para evitar la colonización de H. pilosella debería involucrar la reducción del suelo desnudo. En el norte de Tierra del Fuego se ha observado una asociación entre la historia de disturbios masivos del suelo (p.ej., obras viales, locaciones petroleras, canteras, potreros de aguante, chacras, implantación de pasturas, quemas, etc.) con la ocurrencia actual de parches grandes de la invasora (Cipriotti et al. 2010; Rauber et al. 2012). En el mismo sentido, se reportó una relación estrecha entre la existencia de parches de suelo descubierto y el reclutamiento y colonización a partir de semillas de $H$. pilosella en esos micro-sitios para pastizales templado-fríos de Suiza y Alemania (Winkler \& Stöcklin 2002). Por lo tanto, las obras que impliquen movimientos masivos de suelo deben ir seguidas de re-vegetaciones con especies nativas o naturalizadas para no dejar el suelo expuesto. Asimismo, el manejo del pastoreo a través de por ejemplo la reducción de la carga, la implementación de pastoreos rotativos, o incluso el descanso permanente, debe tener como objetivo evitar tiempos de ocupación prolongados con altas cargas, ya que el pisoteo animal provoca un aumento de la cobertura de suelo desnudo.

En este trabajo presentamos medidas de control a escala local que fueron eficaces para la reducción a mediano plazo de la cobertura de la invasora y de la recuperación de un elenco de especies graminoides e hierbas (tanto nativas como naturalizadas), deseables desde el punto de vista forrajero. La aplicación local de herbicidas selectivos o la combinación de fertilizaciones N-P con la exclusión temporal del pastoreo resultaron en una estrategia de control para contener la invasión de $H$. pilosella en la Estepa Fueguina a mediano plazo. Sin embargo, las medidas de control local aquí propuestas deben integrarse en un plan de manejo de los pastizales, que incluya un manejo del pastoreo de modo de disminuir y revertir su impacto actual sobre la vegetación, un monitoreo regular de la invasión, y la aplicación de técnicas de restauración en áreas con disturbios masivos del suelo. Este conjunto de prácticas debe adaptarse atendiendo las marcadas diferencias que existen en la invasión y su contexto a través de la Estepa Fueguina (Cipriotti et al. 2010).

Agradecimientos. Agradecemos a E. Livraghi, R. Cerezani, V. Canalis, M. Jacob, H. Pico, C. García Sampedro, M.E. Gallego, E. Olmedo y M. Sleiman por el apoyo logístico y la asistencia técnica durante el proyecto. A los productores ganaderos de Tierra del Fuego, en especial al Sr. Errol O’Byrne de Ea. Cullen por su gran predisposición. A los organizadores del simposio de la Reunión Argentina de Ecología 2012 y editores invitados de esta edición especial sobre invasiones en Patagonia, A. Valenzuela y L. Fasola, a M. Semmartin y dos revisores anónimos que con sus aportes permitieron mejorar la versión original. Los trabajos aquí presentados fueron parcialmente solventados por subsidios del CFI, la ANPCyT a través del PICT N²010-00474, y la Universidad de Buenos Aires a través del proyecto UBACyT Nº024.

\section{BiBLIOGRAFÍA}

Aagesen, D. 2000. Crisis and conservation at the end of the world: sheep ranching in Argentine Patagonia. Environmental Conservation, 27:208-215.

Allan, HH. 1924. Notes of the occurrence of certain exotic plants in New Zealand. New Zealand Journal of Agriculture, 29:311-315.

Anchorena, J; A Cingolani; E Livraghi; Mb Collantes \& S StOFFElla. 2001. Manejo del pastoreo de ovejas en Tierra del Fuego. EDIPUBLI S.A. ISBN 987-99049-2-3, Buenos Aires, Argentina.

Bellingham, PJ \& DA Coomes. 2003. Grazing and community structure as determinants of invasion success by scotch broom in a New Zealand montane shrubland. Diversity and Distributions, 9:19-28.

Bishor, GF \& AJ Davy. 1994. Hieracium pilosella L. (Pilosella officinarum F. Schultz and Schultz-Bip.). Journal of Ecology, 82:195-210.

Braun, K. 2009. Efectos del pastoreo, el ambiente y las invasiones sobre procesos de la estepa fueguina. Ph.D. thesis, Universidad de Buenos Aires, Buenos Aires, Argentina.

BROWN, JR \& SR ARCHER. 1989. Woody plant invasion of grasslands: establishment of honey mesquite (Prosopis 
glandulosa var. glandulosa) on sites differing in herbaceous biomass and grazing history. Oecologia, 80:19-26.

Buckland, SM; K Thompson; JG Hodgson \& JP Grime. 2001. Grassland invasions: effects of manipulations of climate and management. Journal of Applied Ecology, 38: 301-309.

Cabeza, S; C Escartin; MB Collantes \& RB Rauber. 2011. Propuesta de manejo para la maleza Hieracium pilosella L. en la Estepa Fueguina. Informe Técnico CFI, Buenos Aires, p. 118.

Cingolani, A; G Posse \& MB Collantes. 2005. Plant functional traits, herbivore selectivity and response to sheep grazing in Tierra del Fuego steppes (Patagonia, Argentina). Journal of Applied Ecology, 42:50-59.

Cipriotti, PA; RB Rauber; MB Collantes; K Braun \& C Escartín. 2010. Hieracium pilosella invasion in the Tierra del Fuego steppe, Southern Patagonia. Biological Invasions, 12:2523-2535.

Cipriotti, PA; RB Rauber; MB Collantes; K Braun \& C EsCARTín. 2012. Control measures for a recent invasion of Hieracium pilosella in Southern Patagonian rangelands. Weed Research, 52:98-105.

Collantes, MB; J Anchorena \& AM Cingolani. 1999. The steppes of Tierra del Fuego: floristic and growth-form patterns controlled by soil fertility and moisture. Plant Ecology, 140:61-75.

Collantes, MB; K Braun, C Escartín, AM Cingolani \& J ANCHORENA. 2005. Patrones de cambio de la vegetación de la estepa fueguina en relación al pastoreo. En: $L a$ heterogeneidad de la vegetación de los agroecosistemas (eds. M Oesterheld, MR Aguiar, CM Ghersa \& JM Paruelo), 235251. Editorial de la Facultad de Agronomía, Universidad de Buenos Aires, Buenos Aires, Argentina.

Davis, MA; JP Grime \& K Thompson. 2000. Fluctuating resources in plant communities: a general theory of invasibility. Journal of Ecology, 88:528-534.

Di Castri, F; RH Groves; FJ Kruger; M Rejmánek; M Williamson; JA DraKe \& HA MoONEY. 1989. Biological Invasions: A Global Perspective. John Wiley \& Sons, p. 550.

DiTomaso, JM. 2000. Invasive weeds in rangelands: species, impacts, and management. Weed Science, 48: 255-265.

DomínguEZ, E. 2004. Plantas exóticas presentes en el Parque Nacional Pali Aike, XII Región, Chile. Chloris Chilensis, 7(2), http://www.chlorischile.cl.

Duncan, RP; KM Colmoun \& BD Foran. 1997. The distribution and abundance of Hieracium species (hawkweeds) in the dry grasslands of Canterbury and Otago. New Zealand Journal of Ecology, 21:51-62.

Firn, J; T Rout; H Possingham \& YM Buckley. 2008. Managing beyond the invader: manipulating disturbance of natives simplifies control efforts. Journal of Applied Ecology, 45:1143-1151.

FirN, J; APN HOUSE \& YM BUCKLEY. 2010. Alternative states models provide an effective framework for invasive species control and restoration of native communities. Journal of Applied Ecology, 47:96-105.

Golluscio, RA; ADeregibus \& JM Paruelo. 1998. Sustainability and range management in the Patagonian steppes. Ecología Austral, 8:265-284.

Grime, JP \& DW JefFrey. 1965. Seedling establishment in vertical gradients of sunlight. Journal of Ecology, 53: 621-642.
Grosskopf, G; LA SMith \& P SYREtT. 2002. Host range of Cheilosia urbana (Meigen) and Cheilosia psilophthalma (Becker) (Diptera: Syrphidae), candidates for the biological control of invasive alien hawkweeds (Hieracium spp., Asteraceae) in New Zealand. Biological Control, 24:7-19.

Hobbs, RJ \& LF HueneKKe. 1992. Disturbance, diversity and invasion: implication for conservation. Conservation Biology, 6:324-337.

Huwer, RK; DT Briese; PM Dowling; DR Kemp; WM LonsDale; DL MichalK; MJ Neave; AW Sheppard \& TL WoodBurn. 2005. Can an integrated management approach provide a basis for long-term prevention of weed dominance in Australian pasture systems? Weed Research, 45:175-192.

Johnstone, PD; JB WiLson \& AG BREMNER. 1999. Change in Hieracium populations in Eastern Otago over the period 1982-1992. New Zealand Journal of Ecology, 23:31-38.

Lamoureaux, SL; D Kelly \& ND Barlow. 2003. Population dynamics in mature stands of Hieracium pilosella in New Zealand. Plant Ecology, 166:263-273.

Livraghi, E; S Cabeza; R Kofalt; G Humano; M Mascó \& L Montes L. 1998. Documento de trabajo sobre Hieracium pilosella L. Informe Técnico INTA.

MACK, RN. 1989. Temperate grasslands vulnerable to plant invasions: characteristics and consequences. In: Biological invasions: a global perspective (eds. JA Drake, HA Mooney, F Di Castri, RH Groves, FJ Kruger, M Rejmánek \& W Williamson), 155-179. John Wiley and Sons, Brisbane, Australia.

Mack, MC; WM Simberloff; H Lonsdale; M Evans; FA Clout \& SD Bazzaz. 2000. Biotic Invasion: Causes, Epidemiology, Global Consequences and Control. Issues in Ecology, 5:1-19.

Makepeace, W. 1985. Growth, reproduction, and production biology of mouse-ear and king devil hawkweed in eastern South Island, New Zealand. New Zealand Journal of Botany, 23:65-78.

MAKEPEACE, W; AT DoBSON \& D SCOTT. 1985. Interference phenomena due to mouse-ear and king-devil hawkweed. New Zealand Journal of Botany, 23:79-90.

Mansilla, HC; TM Cortés; CC Von MoltKe; PB Villalón; DG GuTIÉRREZ \& RP ROJAs. 2011. Programa de control biológico de Hieracium pilosella L. (Compositae: Asteraceae) en la región de Magallanes y Antártica chilena. Informe Técnico SAG, Chile, p. 42.

MeUrK, CD; S WaLKer; RS Gibson \& P Espie. 2002. Changes in vegetation states in grazed and ungrazed Mackenzie Basin grasslands, New Zealand, 1990-2000. New Zealand Journal of Ecology, 26:95-106.

Moen, J \& CD Meurk. 2001. Competitive abilities of three indigenous New Zealand plant species in relation to the introduced plant Hieracium pilosella. Basic and Applied Ecology, 2:243-250.

Norton, DA; PR Espie; W Murray \& J Murray. 2006. Influence of pastoral management on plant biodiversity in a depleted short tussock grassland, Mackenzie Basin. New Zealand Journal of Ecology, 30:335-344.

Paruelo, JM; MB Bertiller; T Schlichter \& FR Coronato. 1993. Secuencias de deterioro en distintos ambientes patagónicos. Su caracterización mediante el modelo de Estados y Transiciones. Proyecto lucha contra la desertificación en Patagonia. (LUDEPA-INTA-GTZ). Bariloche, $110 \mathrm{p}$.

Perelman, SB; RJC León \& JP Bussacca. 1997. Floristic 
changes related to grazing intensity in a Patagonian shrub steppe. Ecography, 20:400-406.

Posse, G; J Anchorena \& MB Collantes. 1996. Seasonal diets of sheep in the steppe region of Tierra del Fuego, Argentina. Journal of Range Management, 49:24-30.

RAUBer, RB; MB COLlantes; PA CipRIOTTI \& J ANCHORENA J. 2012. Biotic and abiotic constraints to a plant invasion in vegetation communities of Tierra del Fuego. Austral Ecology, 38:436-442.

Reynolds, JF; DM StafFord-Smith; EF Lambin; BL TURner II; M Mortimore; SPJ Batterbury et al. 2007. Global Desertification: Building a Science for Dryland Development. Science, 316:847-851.

Rose, AB; KH Platt \& CM Frampton. 1995. Vegetation change over 25 years in a New Zealand short-tussock grassland: Effects of sheep grazing and exotic invasions. New Zealand Journal of Ecology, 19:163-174.

Rose, AB \& CM Frampton. 2007. Rapid short-tussock grassland decline with and without grazing, Marlborough, New Zealand. New Zealand Journal of Ecology, 31:232-244.

Salinas Brescovic, CA. 2002. Eficacia de diferentes herbicidas sobre el crecimiento y desarrollo de Hieracium pilosella L. bajo dos niveles de fertilización nitrogenada en una pradera naturalizada de la Región Magallanes. Tesis de Licenciatura en Agronomía. Universidad Austral de Chile, Valdivia. Chile.

Schlesinger, WH; JF Reynolds; GL Cunningham; LF HUENNEKE; WM JARREL; RA ViRGINIA \& WG WhitFord. 1990. Biological feedbacks in global desertification. Science, 247:1043-1048.

ScotT, D. 1993. Response of Hieracium on two long term manipulative agricultural trials. New Zealand Journal of Ecology, 17:41-46.

SCOTT, D \& BL SUTHERLAND. 1993. Interaction between some pasture species and two Hieracium species. New Zealand
Journal of Ecology, 17:47-51.

SimberLofF, D. 2009. We can eliminate invasions or live with them. Successful management projects. Biological Invasions, 11:149-157.

SORIANO, A. 1983. Deserts and Semideserts of Patagonia. In: West N.E. Temperate Deserts and Semideserts. Elsevier Scientific, Amsterdan. 423-460.

Speziale, KL \& C ezcurRa. 2011. Patterns of alien plant invasions in northwestern Patagonia, argentina. Journal of Arid Environments, 75:890-897.

StraUCh Bertin, O. 2012. Medidas de mitigación y control de Pilosela: Una primera aproximación. Informe técnico INIA Kampenaike, p1-2.

Treskonova, M. 1991. Changes in the structure of tall tussock grasslands and infestation by specie of Hieracium in the Mackenzie country, New Zealand. New Zealand Journal of Ecology, 15:65-78.

Vitousek, PM; Ha Mooney; J Lubchenko \& JM Melillo. 1997. Human domination of Earth Ecosystems. Science, 277:494-499.

Voss, EG \& MW BöHLKe. 1978. The status of certain hawkweeds (Hieracium subgenus Pilosella) in Michigan. The Michigan Botanist, 17:35-47.

WALKER, S; JB WiLsON \& WG LeE. 2005. Does fluctuating resource availability increase invasibility? Evidence from field experiments in New Zealand short tussock grassland. Biological Invasions, 7:195-211.

WinKLER, E \& J STÖCKLIN. 2002. Sexual and vegetative reproduction of Hieracium pilosella L. under competition and disturbance: A grid-based simulation model. Annals of Botany, 89:525-536.

WOODMAN, RF; WL LOWTHER; RP LitTLEJOHN \& RF HORREL. 1997. Establishment response of 12 legumes to nitrogen fertilizer rate and placement when direct drilled into Hieracium-infested, montane tussock grasslands. New Zealand Journal of Agricultural Research, 41:53-63. 